\title{
Anfíbios anuros da RPPN Campo Escoteiro Geraldo Hugo Nunes, Município de Guapimirim, Rio de Janeiro, Sudeste do Brasil
}

\author{
Thiago Silva-Soares ${ }^{1,3}$, Fabio Hepp ${ }^{1}$, Paulo Nogueira Costa ${ }^{1}$, \\ Cyro de Luna-Dias', Márcia dos Reis Gomes ${ }^{1}$, \\ Ana Maria Paulino Telles de Carvalho e Silva ${ }^{2}$ \& Sergio Potsch de Carvalho e Silva ${ }^{1}$ \\ ${ }^{1}$ Laboratótio de Anfíbios e Répteis, Departamento de Zoologia, \\ Instituto de Biologia, Universidade Federal do Rio de Janeiro - UFRJ, \\ Cidade Universitária, CP 68044, CEP 21944-970, Ilha do Fundão, Rio de Janeiro, RJ, Brasil \\ ${ }^{2}$ Laboratório de Biossistemática de Anfíbios, Departamento de Zoologia, Instituto de Biociências, \\ Universidade Federal do Estado do Rio de Janeiro - UNIRIO, \\ Av. Pasteur 458, Bloco III, ECB, Sl.402 - Urca, CEP 22290-240, Rio de Janeiro, RJ, Brasil \\ ${ }^{3}$ Autor para correspondência: Thiago Silva-Soares, e-mail: thiagossoares@ufrj.br
}

SILVA-SOARES, T., HEPP, F., COSTA, P.N., LUNA-DIAS, C., GOMES, M.R., CARVALHO-E-SILVA, A.M.P.T. \& CARVALHO E SILVA, S.P. Anuran Amphibians from RPPN Campo Escoteiro Geraldo Hugo Nunes, Guapimirim Municipality, Rio de Janeiro, Southeastern Brazil. Biota Neotrop. 10(2): http://www. biotaneotropica.org.br/v10n2/en/abstract?inventory+bn01210022010.

\begin{abstract}
We studied the anuran amphibians from RPPN Campo Escoteiro Geraldo Hugo Nunes. The region is located in the Municipality of Guapimirim, State of Rio de Janeiro, in Southeastern Brazil and represents a lowland Atlantic Rainforest remaining, which has 45.2 ha. The hydromorphic soil is conducive to the occurrence of permanent and temporary wetlands. For the study, we carried out field expeditions since the 1980's, being the collected specimens housed at the zoological collection of the Instituto de Biologia, Universidade Federal do Rio de Janeiro. A total of 40 amphibian species of the order Anura have been found at the study site. The species are distributed in 10 families: Hylidae ( $\mathrm{N}=23$ ), Bufonidae (4), Leptodactylidae (4), Cycloramphidae (2), Microhylidae (2), and Brachycephalidae, Craugastoridae, Hemiphractidae, Leiuperidae and Strabomantidae with one species each. Data on local reproductive environments of the recorded species are provided.
\end{abstract}

Keywords: amphibian, anurans, lowland Atlantic Rainforest, conservation status, inventory.

SILVA-SOARES, T., HEPP, F., COSTA, P.N., LUNA-DIAS, C., GOMES, M.R., CARVALHO-E-SILVA, A.M.P.T. \& CARVALHO E SILVA, S.P. Anfíbios anuros da RPPN Campo Escoteiro Geraldo Hugo Nunes, Município de Guapimirim, Rio de Janeiro, Sudeste do Brasil. Biota Neotrop. 10(2): http://www.biotaneotropica.org.br/ v10n2/pt/abstract?inventory+bn01210022010.

Resumo: Os anfíbios anuros da RPPN Campo Escoteiro Geraldo Hugo Nunes, localizado no Município de Guapimirim, Estado do Rio de Janeiro, foram inventariados e estudados. A região é um remanescente de Mata Atlântica de Baixada que ocupa 45,2 ha e está situada sobre um solo hidromórfico que favorece a ocorrência de áreas alagadas permanentes e temporárias. Para o inventário, realizamos excursões para coleta e registro de anfíbios desde a década de 80, estando este material tombado na coleção zoológica do Instituto de Biologia da Universidade Federal do Rio de Janeiro. Um total de 40 espécies de anfíbios da ordem Anura foi encontrado na região, distribuídas em 10 famílias: Hylidae ( $\mathrm{N}=23$ ), Bufonidae (4), Leptodactylidae (4), Cycloramphidae (2), Microhylidae (2), Brachycephalidae, Craugastoridae, Hemiphractidae, Leiuperidae e Strabomantidae com uma espécie cada.

Palavras-chave: anfíbios, anuros, Mata Atlântica de baixada, status de conservação, inventário. 


\section{Introdução}

Estima-se que o Brasil possua aproximadamente $13 \%$ da biota mundial (Lewinsohn \& Prado 2005). Apesar da enorme biodiversidade do país, diversos autores chamam a atenção ao fato de grande parte da fauna brasileira ainda ser desconhecida (Silvano \& Segalla 2005, Rodrigues 2005). Possuindo mais de 875 espécies, o Brasil é o país com a maior diversidade de anfíbios do mundo (Sociedade... 2010), sendo ainda frequente a descrição de novas espécies (mais de 60 nos últimos quatro anos).

Atualmente, o domínio morfoclimático da Mata Atlântica é provavelmente o ecossistema mais ameaçado do mundo. Dos 139.584.893 ha originais, a Mata Atlântica está restrita agora a aproximadamente 16.377 .472 ha, estando a maior parte distribuída em pequenos fragmentos (<100 ha como sugerido por Ranta et al. (1998)) que representam $11,73 \%$ da extensão original (Ribeiro et al. 2009) e que, em grande parte, ainda estão sob enorme pressão antrópica e forte risco de extinção (Morellato \& Haddad 2000).

De acordo com a IUCN (Internacional... 2008a), só no Brasil, 320 espécies de vertebrados se encontram em estado de conservação crítico ou extintas, sendo que 30 destes são anfíbios. Embora a literatura disponha destes números, muitas lacunas de conhecimento deste táxon persistem, tendo várias espécies sido classificadas como "dados deficientes" (196 spp.), além de várias outras cujo status sequer foi avaliado. A coordenação do Global Amphibian Assessment afirma que ainda não foi possível chegar a um consenso, junto aos especialistas, quanto ao status de conservação de algumas espécies endêmicas de anfíbios brasileiros devido à falta de estudos (Internacional... 2008b).

Considerando que os estudos experimentais, de monitoramento e os inventários constituem configuração primária de documentação e entendimento do declínio de anfíbios em todo o mundo
(Biek et al. 2002), a comunidade de anfíbios da RPPN Campo Escoteiro Geraldo Hugo Nunes (CEGHN) e de seus arredores foi inventariada, verificando aspectos do sítio reprodutivo local das espécies encontradas.

A RPPN Campo Escoteiro Geraldo Hugo Nunes é um pequeno representante dos poucos remanescentes de vegetação de Mata Atlântica de baixada. Alguns estudos relacionados à flora e à fauna como em aves, peixes e insetos já foram realizados na região (Costa \& Lacerda 1988, Guedes 1988, Carvalho 1989, Costa 2002), porém, um estudo mais abrangente acerca da comunidade de anfíbios nunca foi publicado.

Aqui apresentamos a lista de espécies de anfíbios anuros de um remanescente da Mata Atlântica do Sudeste do Brasil, a Reserva Particular de Patrimônio Natural Campo Escoteiro Geraldo Hugo Nunes.

\section{Material e Métodos}

\section{1. Área de estudo}

A RPPN Campo Escoteiro Geraldo Hugo Nunes (CEGHN), uma área de 45.2 ha pertencente à Associação União dos Escoteiros do Brasil, está localizada no distrito de Citrolândia, Município de Guapimirim, sopé da Serra dos Órgãos, latitude entre $22^{\circ} 34^{\prime} 33^{\prime \prime} \mathrm{S}-22^{\circ} 35^{\prime} 05^{\prime \prime} \mathrm{S}$ e $43^{\circ} 01^{\prime} 44^{\prime \prime} \mathrm{O}-43^{\circ} 02^{\prime} 25^{\prime \prime} \mathrm{O}$. A área recoberta por mata apresenta relevo plano com pequenas elevações com cota máxima de $48 \mathrm{~m}$ de altitude e possui a forma de um istmo alongado outrora ligado à mata que recobre o vizinho maciço da Serra dos Órgãos (Figura 1).

A área em questão, que já foi uma fazenda de propriedade particular, possui sua formação vegetal inalterada desde 1968,

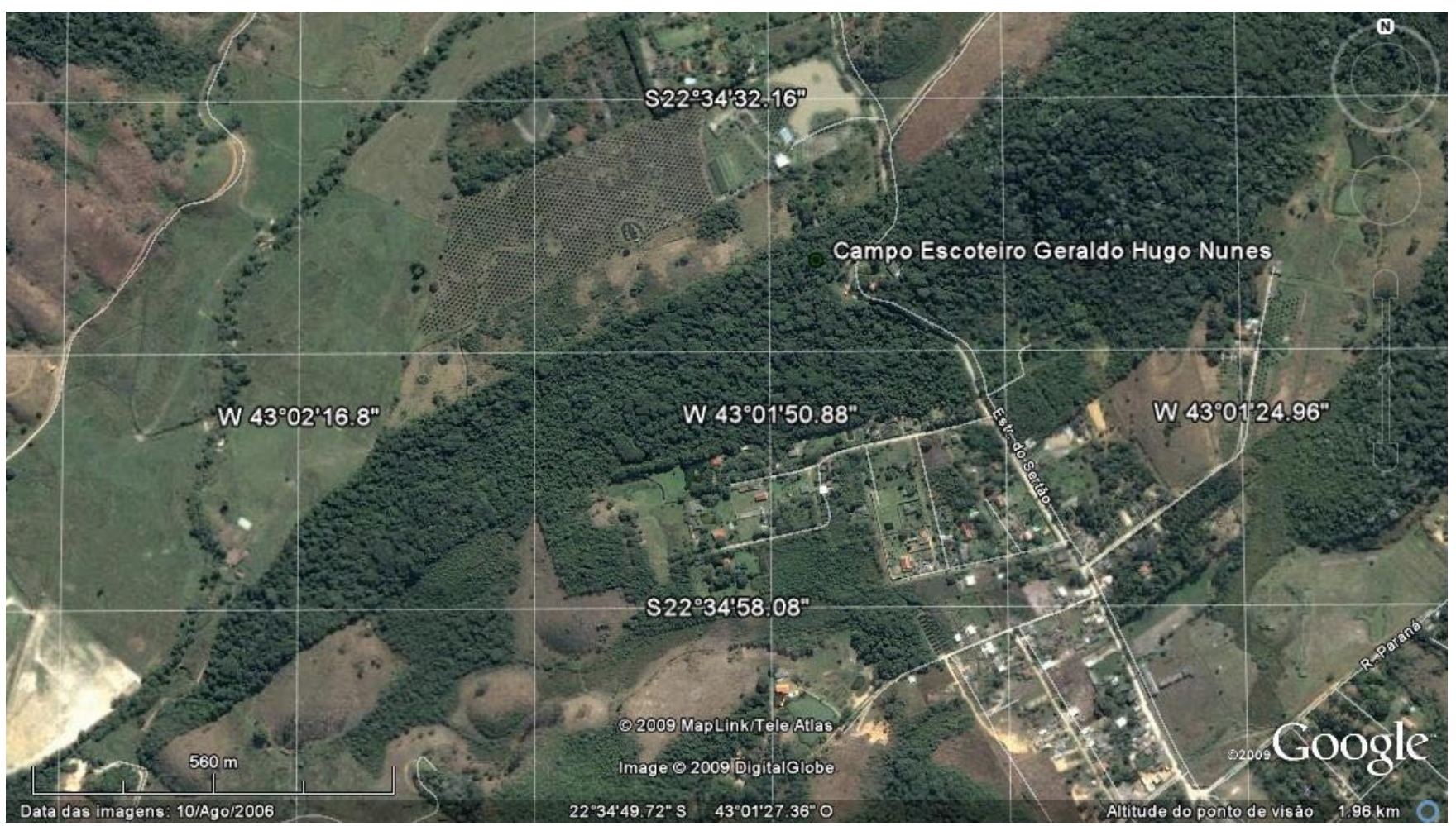

Figura 1. Localização da RPPN Campo Escoteiro Geraldo Hugo Nunes, Município de Guapimirim, Rio de Janeiro, Sudeste do Brasil.

Figure 1. Location of RPPN Campo Escoteiro Geraldo Hugo Nunes, Guapimirim Municipality, Rio de Janeiro, Southeastern Brazil. 
data em que foi adquirida pela União dos Escoteiros do Brasil (2007) e, posteriormente, transformada em Reserva Particular do Patrimônio Natural - RPPN Geraldo Hugo Nunes, criada em 14 de novembro de 2008 com o desígnio de contribuir com a preservação da biodiversidade da Mata Atlântica fluminense em benefício das gerações atuais e futuras (Instituto... 2008), com uma área de 20,3 ha, correspondendo à extremidade do istmo. Regiões que circundam a área de estudo também foram inventariadas devido ao fato de possuírem condições ambientais características para reprodução de anuros, tendo destaque para um sítio de piscicultura e suas proximidades. Assim, considerando o CEGHN juntamente com a área que o circunda, a área total de estudo abrange aproximadamente 70 ha.

\subsection{Clima e solo}

Segundo Bernardes (1952), o clima da região é quente e úmido, sem estação seca, correspondendo ao tipo Af da classificação de Köppen. As normais climatológicas calculadas a partir de observações feitas na estação meteorológica de Citrolândia (22 $36^{\circ}$ ' S e $43^{\circ} 02^{\prime} \mathrm{O}$; $15 \mathrm{~m}$ de altitude), indicam temperatura média anual é de $21,9^{\circ} \mathrm{C}$, sendo janeiro o mês mais quente $\left(25,3^{\circ} \mathrm{C}\right)$ e julho o mês mais frio $\left(17,9{ }^{\circ} \mathrm{C}\right)$ e precipitação abundante com total anual de $2050 \mathrm{~mm}$, variando as médias mensais de $337,8 \mathrm{~mm}$ (fevereiro) a $59,3 \mathrm{~mm}$ (julho). Os meses de menor pluviosidade são os de maio a outubro, que correspondem aos meses mais frios.

O trecho estudado ocorre sobre um solo arenoso, hidromórfico, extremamente ácido e recoberto por densa camada de serrapilheira que tornam o terreno periodicamente encharcado, por ocasião das chuvas, e elevam o teor de matéria orgânica disponível (Guedes 1988).

\subsection{Vegetação}

A floresta do CEGHN está inserida no domínio morfoclimático da Mata Atlântica (Ab'Saber 1977) e apresenta-se como uma mata densa, com caules eretos sem raízes tubulares, onde há grande variedade de samambaias, orquídeas e bromélias epífitas (Guedes 1988). Estas características associadas ao tipo de solo permitem a caracterização da mata como Mata Pluvial Tropical de Planícies Costeiras (Hueck 1972) ou simplesmente como Mata de Baixada (Eiten 1983). As árvores que compõem o estrato arbóreo são relativamente baixas, com altura média em torno de $7 \mathrm{~m}$, onde se destacam as árvores emergentes de até $20 \mathrm{~m}$ (Guedes 1988). Entretanto a vegetação encontra-se muito perturbada por atividades humanas possuindo cobertura rala e subbosque denso composto principalmente por arbustos.

\section{Amostragem}

Desde novembro de 1983 foram realizados estudos de campo para coleta e observação dos anfíbios na área de estudo, nas diversas estações do ano, possibilitando assim, observar o ambiente reprodutivo da maioria das espécies na região.

Para a coleta, o método utilizado foi captura manual por busca ativa visual e auditiva, durante os períodos noturno e diurno. Os espécimes coletados nas expedições recentes foram fotografados e fixados de acordo com as técnicas usuais e atualmente válidas para o grupo (Calleffo 2002).

Durante a busca ativa, troncos, pedras, ocos de árvores, bromélias e serrapilheira foram revirados e/ou vasculhados além dos locais com corpos d'água permanentes ou temporários, como brejos e poças. Outros locais de interior e borda de mata sem corpos d'água também foram amostrados objetivando a captura de espécies que depositam seus ovos no solo úmido e que são frequentemente encontradas na serrapilheira.
Foram classificados sete ambientes reprodutivos das espécies de anuros, sendo estes: Brejos (alagados em área aberta sem conexão com corpos de água corrente), serrapilheira (folhas mortas acumuladas no chão em interior de mata), poça de borda de mata (alagados em borda de mata sem conexão com corpos de água corrente), poça de interior de mata (alagados temporários ou permanentes de interior de mata sem conexão com corpos de água corrente), bromélias, riacho de interior da mata (córrego da água permanente) e um lago permanente formado pela escavação de uma pedreira, atualmente desativada.

O material testemunho (Apêndice 1) encontra-se depositado na coleção de anfíbios do Instituto de Biologia da Universidade Federal do Rio de Janeiro (ZUFRJ).

Para a obtenção dos registros sonoros foram utilizados os gravadores Sony WM-D6C K7 recorder e Marantz PMD 670 digital recorder, acoplados aos microfones unidirecionais Sennheiser ME 67 ou Sennheiser ME 66. As gravações foram depositadas na coleção de vocalizações do Laboratório de Anfíbios e Répteis da Universidade Federal do Rio de Janeiro. Os cantos gravados foram comparados com cantos já descritos e com cantos anteriormente armazenados na coleção supracitada para fins de identificação.

Adicionalmente, foram consultadas a coleção de anfíbios da Universidade Federal do Estado do Rio de Janeiro (UNIRIO), a coleção Eugenio Izecksohn da Universidade Federal Rural do Rio de Janeiro (EI) e a coleção de anfíbios do Museu Nacional (MNRJ).

\section{Resultados e Discussão}

Ao todo, 40 espécies de anfíbios da ordem Anura foram encontradas na RPPN Campo Escoteiro Geraldo Hugo Nunes, estando distribuídas em 10 famílias (Figuras 2 a 6). A família Hylidae foi a mais representativa com 23 espécies em sete gêneros, seguida por Bufonidae (quatro spp., dois gêneros), Leptodactylidae (quatro spp., um gênero), Cycloramphidae e Microhylidae (duas spp., dois gêneros) e Brachycephalidae, Craugastoridae, Hemiphractidae, Leiuperidae e Strabomantidae (uma espécie cada: Tabela 1).

As consultas às coleções de anfíbios do MNRJ, UNIRIO, UFRRJ, proporcionaram a adição de uma única espécie de anuro que não fora encontrado por nós na área de estudo: Dendropsophus anceps. Com exceção desta espécie, nenhuma outra foi encontrada nas coleções científicas citadas, que já não estivessem inclusas no presente trabalho.

No CEGHN Rhinella hoogmoedi (Figura 2a) apresenta cor críptica com a serrapilheira, local que habitam, e está associada ao ambiente reprodutivo de riacho de água límpida, rasa, de leve correnteza e de interior de mata, onde geralmente vocaliza na margem. Entretanto, na RPPN CEGHN, alguns espécimes foram encontrados vocalizando empoleirados em brejaúvas a mais de dois metros de altura. Esta palmeira acumula folhas secas caídas de outras plantas em seu caule por meio dos acúleos (projeções epidérmicas espinescentes) aparentemente propiciando maior estrato espacial e camuflagem para a espécie.

A espécie Haddadus binotatus (Figura 2f), comumente encontrado na região, possui ampla distribuição ocorrendo do Sul da Bahia ao Rio Grande do Sul (Internacional... 2009). De acordo com a IUCN (Internacional... 2009), a espécie se encontra na categoria de não ameaçada devido à sua ampla distribuição, à sua tolerância a ambientes degradados e por suas populações não demonstrarem indícios de declínio. Contudo, no Rio Grande do Sul, H. binotatus se encontra incluída na lista de fauna ameaçada (Vinciprova \& Garcia 2002).

Euparkerella cochranae (Figura 6a) possui tamanho diminuto e é endêmica do Estado do Rio de Janeiro, com poucos locais conhecidos sobre sua ocorrência. A espécie, que tem como localidade tipo a subsede do Parque Nacional da Serra dos Órgãos, também no 

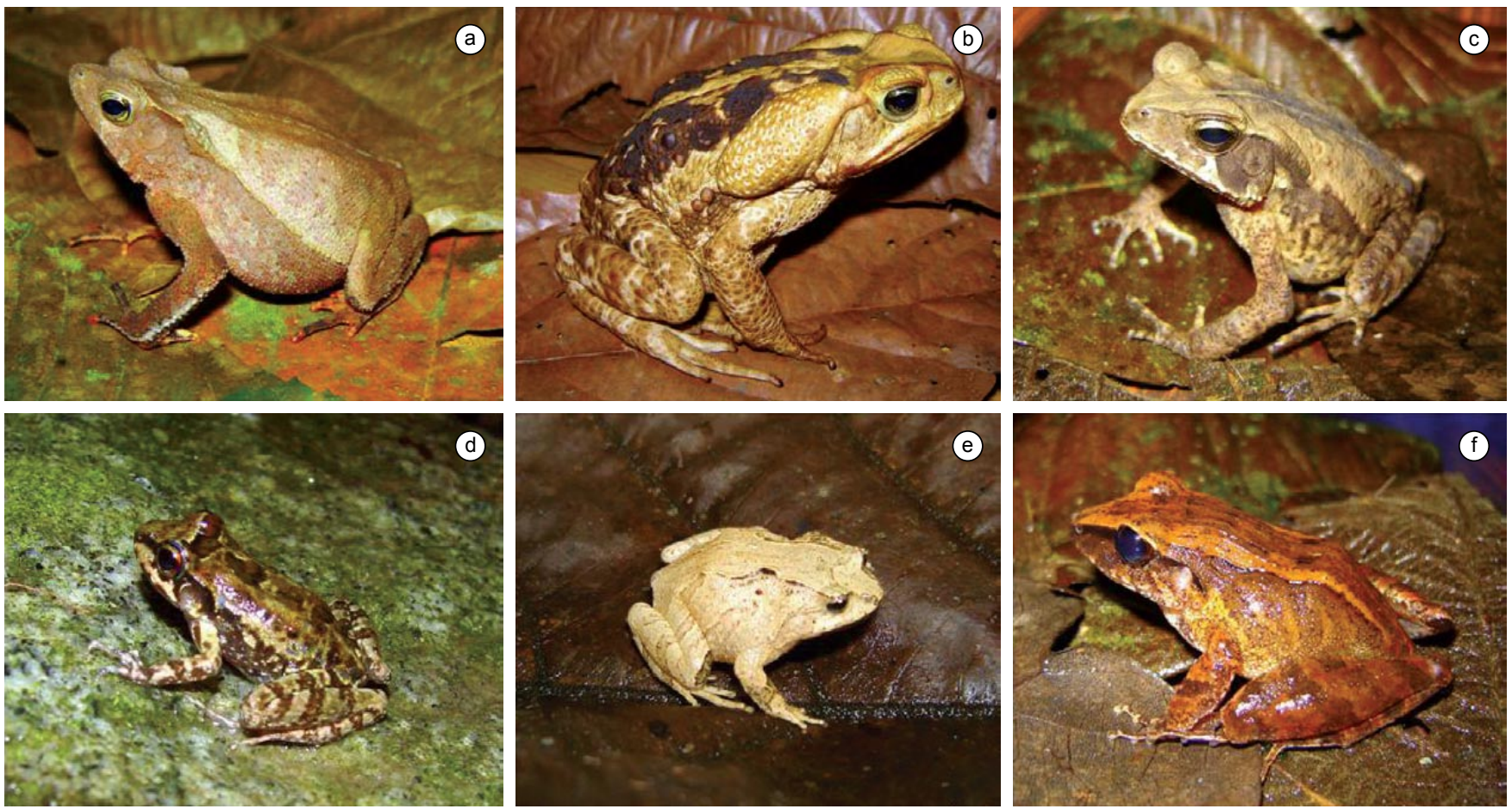

Figura 2. Anuros do Campo Escoteiro Geraldo Hugo Nunes, Município de Guapimirim, Rio de Janeiro: a) Rhinella hoogmoedi; b) Rhinella icterica; c) Rhinella ornata; d) Thoropa miliaris; e) Zachaenus parvulus; and f) Haddadus binotatus.

Figure 2. Anurans from RPPN Campo Escoteiro Geraldo Hugo Nunes, Guapimirim Municipality, Rio de Janeiro: a) Rhinella hoogmoedi; b) Rhinella icterica; c) Rhinella ornata; d) Thoropa miliaris; e) Zachaenus parvulus; f) Haddadus binotatus.
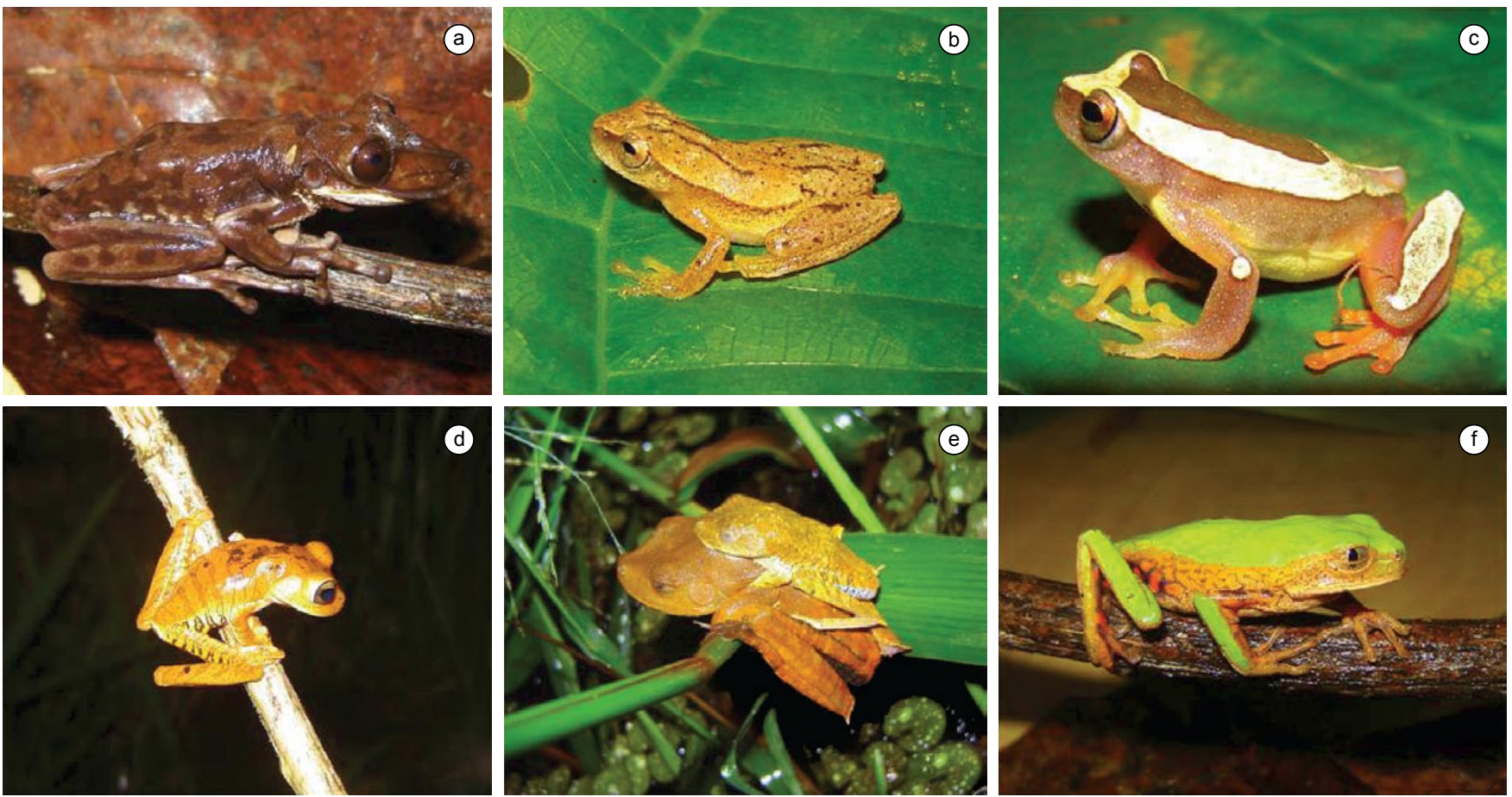

Figura 3. Anuros da RPPN Campo Escoteiro Geraldo Hugo Nunes, Município de Guapimirim, Rio de Janeiro: a) Aparasphenodon brunoi; b) Dendropsophus pseudomeridianus; c) Dendropsophus elegans; d) Hypsboas faber; e) Hypsiboas semilineatus; f) Phyllomedusa rodhei.

Figure 3. Anurans from RPPN Campo Escoteiro Geraldo Hugo Nunes, Guapimirim Municipality, Rio de Janeiro: a) Aparasphenodon brunoi; b) Dendropsophus pseudomeridianus; c) Dendropsophus elegans; d) Hypsboas faber; e) Hypsiboas semilineatus; f) Phyllomedusa rodhei. 

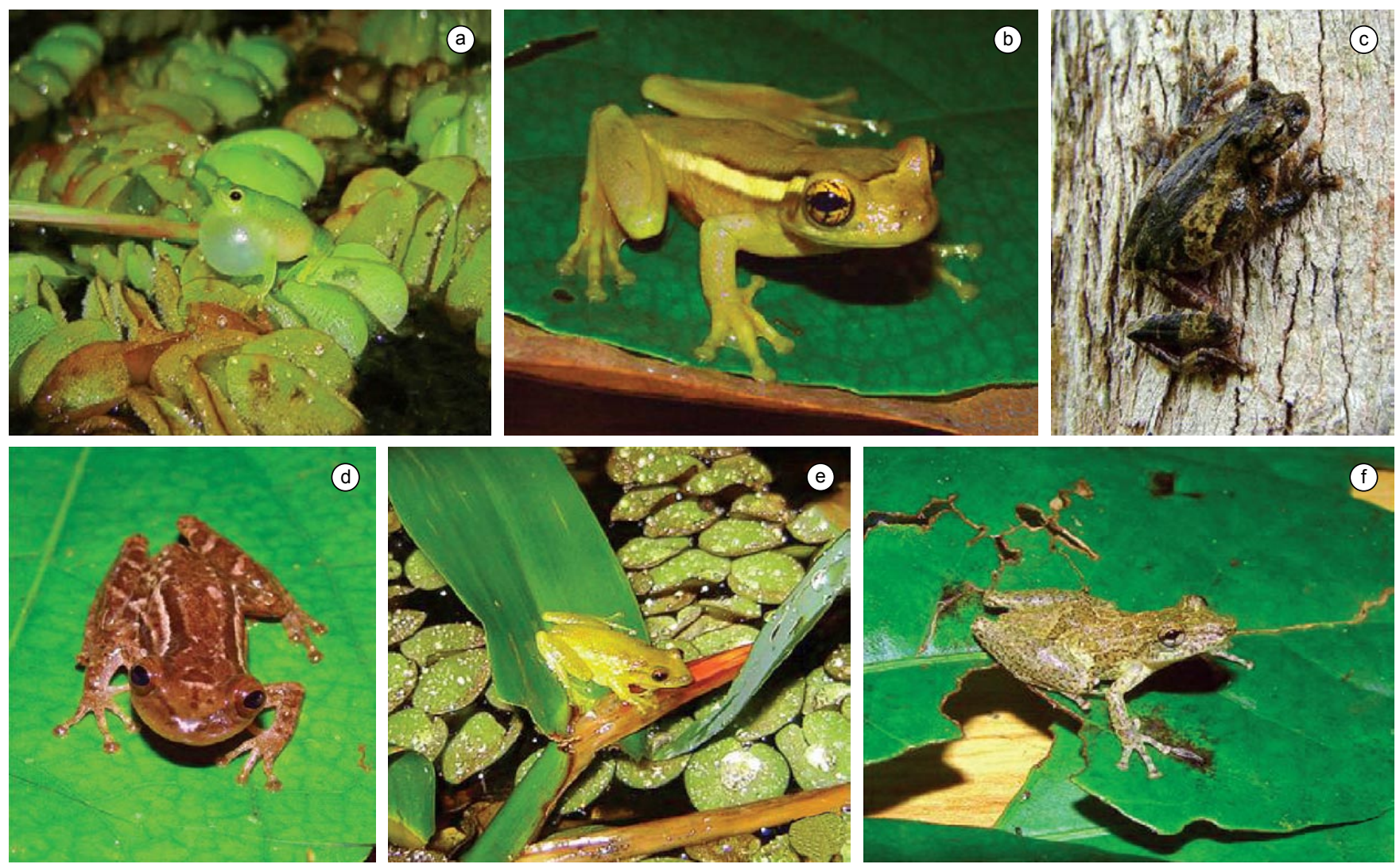

Figura 4. Anuros da RPPN Campo Escoteiro Geraldo Hugo Nunes, Município de Guapimirim, Rio de Janeiro: a) Sphaenorhynchus planicola; b) Trachycephalus mesophaeus; c) Dendropsophus seniculus; d) Scinax cuspidatus; e) Scinax alter; f) Scinax argyreornatus.

Figure 4. Anurans from RPPN Campo Escoteiro Geraldo Hugo Nunes, Guapimirim Municipality, Rio de Janeiro: a) Sphaenorhynchus planicola; b) Trachycephalus mesophaeus; c) Dendropsophus seniculus; d) Scinax cuspidatus; e) Scinax alter; f) Scinax argyreornatus.
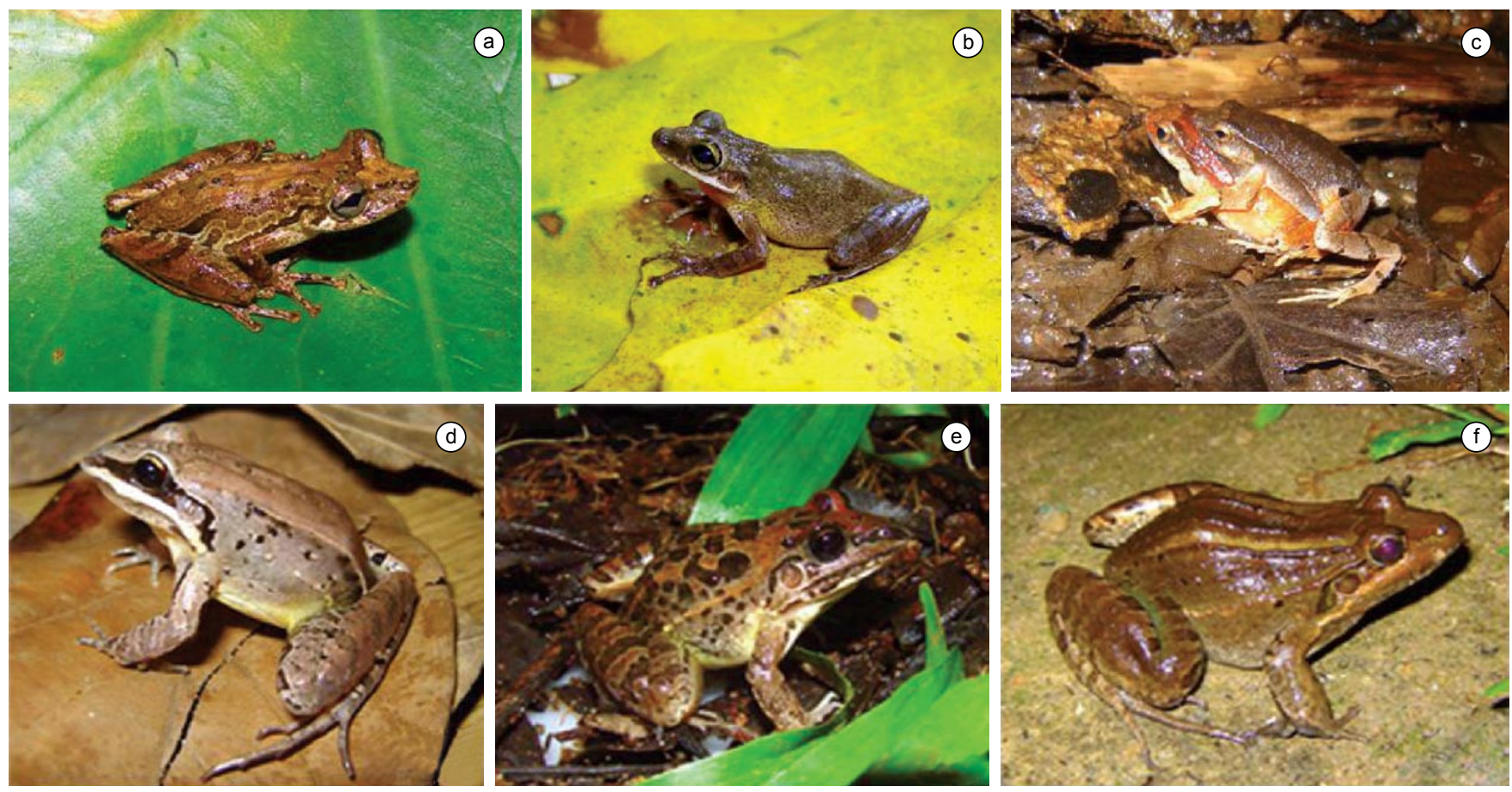

Figura 5. Anuros da RPPN Campo Escoteiro Geraldo Hugo Nunes, Município de Guapimirim, Rio de Janeiro: a) Scinax humilis; b) Scinax similis; c) Physalaemus signifer; d) Leptodactylus spixi; e) Leptodactylus fuscus; f) Leptodactylus latrans.

Figure 5. Anurans from RPPN Campo Escoteiro Geraldo Hugo Nunes, Guapimirim Municipality, Rio de Janeiro: a) Scinax humilis; b) Scinax similis; c) Physalaemus signifer; d) Leptodactylus spixi; e) Leptodactylus fuscus; f) Leptodactylus latrans. 
Tabela 1. Lista das espécies de anfíbios encontrados na RPPN Campo Escoteiro Geraldo Hugo Nunes, Município de Guapimirim, Rio de Janeiro. Abreviações e fonte: Brejos (Bre), serrapilheira (S), poça de borda de mata (PB), poça de interior de mata (PI), bromélias (Bro), riacho de interior da mata (RM) e uma pedreira desativada (P). * Status de Conservação de acordo com Stuart et al. (2008) e IUCN (Internacional... 2009).

Table 1. List of the Amphibian species recorded at RPPN Campo Escoteiro Geraldo Hugo Nunes, Guapimirim Municipality, Rio de Janeiro. Abbreviations and sources: Swamps (Bre), litter (S), pound on forest edge (PB), pound inside forest (PI), bromeliads (Bro), stream inside forest (RM) and an inactive quarry (P). *Conservation status according to Stuart et al. (2008) and IUCN (Internacional... 2009).

\begin{tabular}{|c|c|c|c|c|c|}
\hline Família e espécies & Adulto & Girino & Vocalização & $\begin{array}{c}\text { Status de } \\
\text { conservação* }\end{array}$ & $\begin{array}{c}\text { Habitat reprodutivo } \\
\text { observado }\end{array}$ \\
\hline \multicolumn{6}{|l|}{ BRACHYCEPHALIDAE } \\
\hline Brachycephalus didactylus (Izecksohn, 1971) & $\mathrm{X}$ & - & - & Fora de Risco & $S$ \\
\hline \multicolumn{6}{|l|}{ BUFONIDAE } \\
\hline Dendrophryniscus brevipollicatus Jiménez de la Espada, 1870 & $\mathrm{X}$ & - & - & Fora de Risco & Bro \\
\hline Rhinella icterica (Spix, 1824) & $\mathrm{X}$ & - & - & Fora de Risco & PB, Bre \\
\hline Rhinella hoogmoedi Caramashi \& Pombal 2006 & $\mathrm{X}$ & $\mathrm{X}$ & $\mathrm{X}$ & Fora de Risco & RM \\
\hline Rhinella ornata (Spix, 1824) & $\mathrm{X}$ & $\mathrm{X}$ & $\mathrm{X}$ & Fora de Risco & PB, Bre \\
\hline \multicolumn{6}{|l|}{ CRAUSGASTORIDAE } \\
\hline Haddadus binotatus (Spix, 1824) & $\mathrm{X}$ & - & - & Fora de Risco & $S$ \\
\hline \multicolumn{6}{|l|}{ CYCLORAMPHIDAE } \\
\hline Thoropa miliaris (Spix, 1824) & $\mathrm{X}$ & $X$ & $X$ & Fora de Risco & $\mathrm{P}$ \\
\hline Zachaenus parvulus (Girard, 1853) & $X$ & - & - & Fora de Risco & $\mathrm{S}$ \\
\hline \multicolumn{6}{|l|}{ HEMIPHRACTIDAE } \\
\hline Flectonotus goeldii (Boulenger, 1895) & $\mathrm{X}$ & - & $\mathrm{X}$ & Fora de Risco & Bro \\
\hline \multicolumn{6}{|l|}{ HYLIDAE } \\
\hline Aparasphenodon brunoi Miranda-Ribeiro, 1920 & $\mathrm{X}$ & - & - & Fora de Risco & $\mathrm{PB}$ \\
\hline Dendropsophus anceps (Lutz, 1929) & $\mathrm{X}$ & $\mathrm{X}$ & $\mathrm{X}$ & Fora de Risco & $\mathrm{PB}$ \\
\hline Dendropsophus berthalutzae (Bokermann, 1962) & $X$ & $X$ & $X$ & Fora de Risco & PI \\
\hline Dendropsophus bipunctatus (Spix ,1824) & $\mathrm{X}$ & $\mathrm{X}$ & $\mathrm{X}$ & Fora de Risco & Bre \\
\hline Dendropsophus elegans (Wied-Neuwied, 1824) & $\mathrm{X}$ & $\mathrm{X}$ & $\mathrm{X}$ & Fora de Risco & Bre \\
\hline Dendropsophus giesleri (Mertens, 1950) & $X$ & $X$ & $\mathrm{X}$ & Fora de Risco & $\mathrm{PB}, \mathrm{Bre}$ \\
\hline Dendropsophus meridianus (Lutz, 1954) & $\mathrm{X}$ & $\mathrm{X}$ & $\mathrm{X}$ & Fora de Risco & $\mathrm{PB}$ \\
\hline Dendropsophus minutus (Peters, 1872) & $\mathrm{X}$ & $\mathrm{X}$ & $\mathrm{X}$ & Fora de Risco & PB, Bre \\
\hline Dendropsophus pseudomeridianus (Cruz, Caramaschi \& Dias, 2000) & $\mathrm{X}$ & $\mathrm{X}$ & $\mathrm{X}$ & Fora de Risco & Bre \\
\hline Dendropsophus seniculus (Cope, 1868) & $\mathrm{X}$ & $\mathrm{X}$ & $\mathrm{X}$ & Fora de Risco & Bre \\
\hline Hypsiboas albomarginatus (Spix, 1824) & $\mathrm{X}$ & $\mathrm{X}$ & $\mathrm{X}$ & Fora de Risco & Bre \\
\hline Hypsiboas faber (Wied-Neuwied, 1821) & $\mathrm{X}$ & $X$ & $\mathrm{X}$ & Fora de Risco & Bre \\
\hline Hypsiboas semilineatus (Spix,1824) & $\mathrm{X}$ & $\mathrm{X}$ & $\mathrm{X}$ & Fora de Risco & Bre \\
\hline Phyllomedusa rohdei Mertens, 1926 & $\mathrm{X}$ & $\mathrm{X}$ & $\mathrm{X}$ & Fora de Risco & PB \\
\hline Scinax alter (B. Lutz, 1973) & $X$ & $X$ & $\mathrm{X}$ & Fora de Risco & Bre \\
\hline Scinax aff. alter & $\mathrm{X}$ & $\mathrm{X}$ & $\mathrm{X}$ & - & Bre \\
\hline Scinax argyreornatus (Miranda-Ribeiro, 1926) & $\mathrm{X}$ & $\mathrm{X}$ & $\mathrm{X}$ & Fora de Risco & PI \\
\hline Scinax eurydice (Bokermann, 1968) & $\mathrm{X}$ & $X$ & $X$ & Fora de Risco & $\mathrm{PB}, \mathrm{Bre}$ \\
\hline Scinax cuspidatus (Lutz, 1925) & $\mathrm{X}$ & $X$ & $X$ & Fora de Risco & Bre \\
\hline Scinax humilis (B. Lutz, 1954) & $\mathrm{X}$ & $\mathrm{X}$ & $\mathrm{X}$ & Fora de Risco & PB, Bre \\
\hline Scinax similis (Cochran, 1952) & $X$ & $\mathrm{X}$ & $X$ & Fora de Risco & $\mathrm{PB}, \mathrm{Bre}$ \\
\hline Sphaenorhynchus planicola (Lutz \& Lutz, 1938) & $\mathrm{X}$ & $\mathrm{X}$ & $\mathrm{X}$ & Fora de Risco & Bre \\
\hline Trachycephalus mesophaeus (Hensel, 1867) & $\mathrm{X}$ & $\mathrm{X}$ & $\mathrm{X}$ & Fora de Risco & Bre \\
\hline \multicolumn{6}{|l|}{ LEIUPERIDAE } \\
\hline Physalaemus signifer (Girard, 1853) & $\mathrm{X}$ & $\mathrm{X}$ & $X$ & Fora de Risco & PI \\
\hline \multicolumn{6}{|l|}{ LEPTODACTYLIDAE } \\
\hline Leptodactylus fuscus (Schneider, 1799) & $\mathrm{X}$ & - & $\mathrm{X}$ & Fora de Risco & Bre \\
\hline Leptodactylus latrans (Linnaeus, 1758) & $\mathrm{X}$ & $\mathrm{X}$ & $\mathrm{X}$ & Fora de Risco & Bre \\
\hline Leptodactylus marmoratus (Steindachner, 1867) & $\mathrm{X}$ & - & $\mathrm{X}$ & Fora de Risco & $S$ \\
\hline Leptodactylus spixi Heyer, 1983 & $\mathrm{X}$ & $\mathrm{X}$ & $\mathrm{X}$ & Fora de Risco & PB \\
\hline \multicolumn{6}{|l|}{ MICROHYLIDAE } \\
\hline Myersiella microps (Duméril \& Bibron, 1841) & $\mathrm{X}$ & - & $X$ & Fora de Risco & $\mathrm{S}$ \\
\hline Chiasmocleis carvalhoi Cruz, Caramaschi, \& Izecksohn, 1997 & $\mathrm{X}$ & $\mathrm{X}$ & $\mathrm{X}$ & Em Perigo & PI \\
\hline \multicolumn{6}{|l|}{ STRABOMANTIDAE } \\
\hline Euparkerella cochranae Izecksohn, 1988 & $\mathrm{X}$ & - & $\mathrm{X}$ & Fora de Risco & $\mathrm{S}$ \\
\hline
\end{tabular}



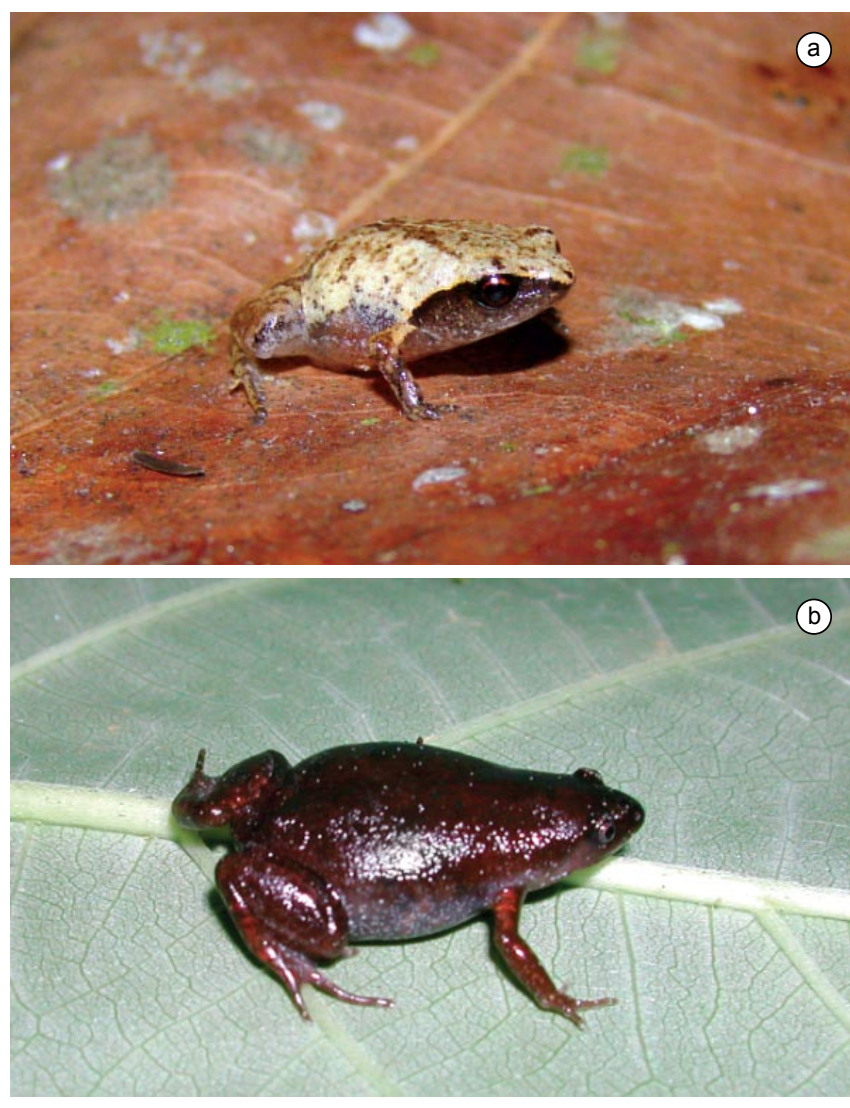

Figura 6. Anuros da RPPN Campo Escoteiro Geraldo Hugo Nunes, Município de Guapimirim, Rio de Janeiro: a) Euparkerella cochranae; b) Chiasmocleis carvalhoi.

Figure 6. Anurans from RPPN Campo Escoteiro Geraldo Hugo Nunes, Guapimirim Municipality, Rio de Janeiro: a) Euparkerella cochranae; b) Chiasmocleis carvalhoi.

Município de Guapimirim, é comum na região principalmente dentro dos limites da área da RPPN onde há serrapilheira acumulada.

A espécie Scinax aff. alter, não pode ser identificada ao seu nível específico pois, apesar de ser morfologicamente semelhante a espécie Scinax alter descrita por A. Lutz (1925) de Manguinhos, bairro da cidade do Rio de Janeiro, e ainda habitar o mesmo ambiente que S. alter no CEGHN, apresenta diferenças morfológicas e acústicas que indicam ser uma espécie provavelmente ainda não descrita. Todavia, apenas a partir de mais estudos poder-se-á definir se esta é realmente uma espécie nova.

De todas as espécies que ocorrem no CEGHN, de acordo com a Lista Vermelha da IUCN (Internacional... 2009) e Stuart et al. (2008), apenas Chiasmocleis carvalhoi (Figura 6b) não se encontra 'Fora de Risco' (Least Concern), sendo esta assinalada como 'Em Perigo' (Endangered) com a afirmação que sua área de ocupação é menor que $500 \mathrm{~km}^{2}$, possuindo distribuição muito fragmentada e que a extensão de seu habitat está provavelmente declinando. Entretanto, além de ser comumente encontrada no CEGHN, a espécie tem sido encontrada em outras regiões do Rio de Janeiro (Carvalho-e-Silva et al. 2008, Salles et al. 2009) e de outros estados como Bahia (Pimenta et al. 2002) e Espírito Santo (Silva-Soares et al. 2009).

A Figura 7 mostra a relação da utilização de ambiente de reprodução por espécies de anuros na área de estudo, sendo que as espécies Rhinella icterica, R. ornata, D. giesleri, D. minutus, $S$. humilis e $S$. similis utilizam mais de um ambiente reprodutivo (ver Tabela 1).

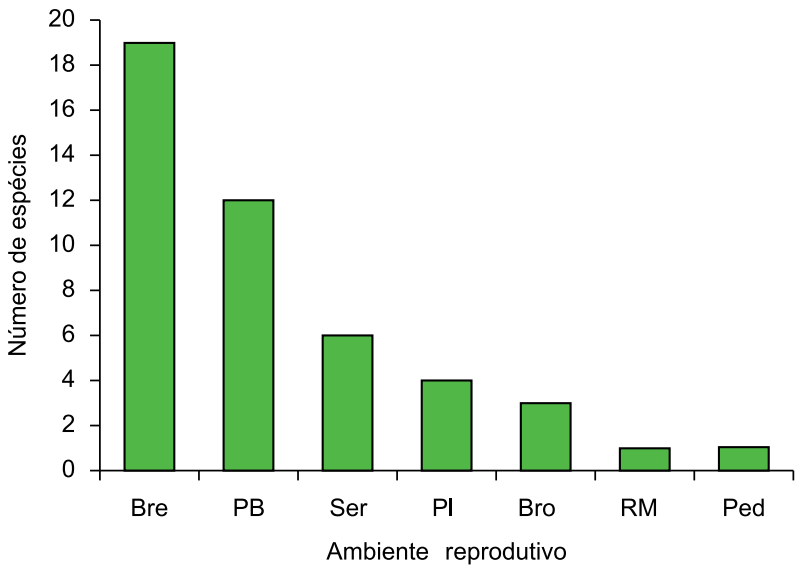

Figura 7. Relação entre o número de espécies por utilização de ambiente reprodutivo. Brejos (Bre), serrapilheira (S), poça de borda de mata (PB), poça de interior de mata (PI), bromélias (Bro), riacho de interior da mata (RM) e uma pedreira desativada $(\mathrm{P})$.

Figure 7. Relation between the number of species per reproductive environment used. Swamps (Bre), litter (S), pound on forest edge (PB), pound inside forest (PI), bromeliads (Bro), stream inside forest (RM) and an inactive quarry $(\mathrm{P})$.

Apesar de pequena, a área de estudo apresenta uma fauna rica em anuros, ou seja, o CEGHN possui aproximadamente $10 \%$ das espécies da Mata Atlântica. O solo de drenagem deficiente possibilita a formação de trechos alagadiços e trechos permanentemente encharcados nas cotas mais baixas do terreno (característica da maior parte do terreno da área de estudo), prejudicando a diversidade de vários outros grupos de flora e fauna (Guedes 1988, L. P. Gonzaga, dados não publicados), ao mesmo tempo em que propicia esta grande riqueza de anuros local, já que a maioria das espécies da região depende desses tipos de ambientes para reprodução.

\section{Agradecimentos}

Agradecemos a todos que contribuíram na organização das excursões e nas coletas de anfíbios na RPPN Campo Escoteiro Geraldo Hugo Nunes. Agradecemos à União dos Escoteiros do Brasil, Região do Rio de Janeiro, pela permissão concedida para a realização desta pesquisa na área durante todos esses anos. Ao Prof. Dr. José Pombal Jr. por permitir o acesso à coleção de anfíbios do Museu Nacional. Ao Rodrigo Salles e Marcos Bilate pela ajuda no Museu Nacional e em campo na RPPN CEGHN. Ao Fábio, funcionário do CEGHN pelo auxílio concedido durante nossa estadia. Agradecemos a Michael Presto pela revisão do inglês e à Fundação de Amparo à Pesquisa do Rio de Janeiro (FAPERJ) e à Coordenação de Aperfeiçoamento de Pessoal de Nível Superior (CAPES) pelo suporte financeiro.

\section{Referências Bibliográficas}

AB'SABER, A.N. 1977. Os domínios morfoclimáticos América do Sul. Geomorfologia 52:1-20.

BIEK, R., FUNK, W.C., MAXELL, B.A. \& MILLS, L.S. 2002. What is missing in amphibian decline research: insights from ecological sensitivity analysis. Conserv. Biol. 16(3):728-734.

BERNARDES, L.M.C. 1952. Tipos de clima do estado do Rio de Janeiro. Rev. Bras. Geog. 14(1):57-80.

CALLEFFO, M.E.V. 2002. Anfíbios. In AURICCHIO, P. \& SALOMÃO, M.G. Técnicas de coleta e preparação de vertebrados para fins científicos e didáticos. Instituto Pau Brasil de História Natural, São Paulo, p.43-74. 
CARVALHO, A.L. 1989. Description of the larva of Neuraeschna costalis (Burmeister), with notes on its biology, and a key to the genera of Brazilian Aeshnidae larvae (Anisoptera). Odonatologica 18(4):325-332.

CARVALHO-E-SILVA, A.M.P.T., SILVA, G.R. \& CARVALHO-E-SILVA, S.P.C.S. 2008. Anuros da Reserva Rio das Pedras, Mangaratiba, RJ, Brasil. Biota Neotrop. 8(1):200-209: http://www.biotaneotropica.org.br/v8n1/pt/ abstract?inventory+bn02608012008 (último acesso em 20/10/1009).

COSTA, W.J.E.M. 2002. Leptolebias marmoratus (Cyprinodontiformes: Rivulidae: Cynolebiatinae): rediscovery and redescription of a rare, miniaturized forest dwelling seasonal fish from southeastern Brazil. Ichthyol. Expl. Freshw. 13(4):379-384.

COSTA, W.J.E.M. \& LACERDA, M.T.C. 1988. Identité et redescription de Cynolebias sandrii et de Cynolebias fluminensis (Cyprinodontiformes, Rivulidae). Rev. Franç. Aquar. Herpet 14:127-132.

EITEN, G. 1983. Classificação da vegetação do Brasil. Conselho Nacional de Desenvolvimento Científico e Tecnológico, Brasília.

GUEDES, R.R. 1988. Composição florística de um trecho de mata perturbada de baixada no Município de Magé, Rio de Janeiro. Arq. Jard. Bot. Rio de Janeiro 29:155-200.

HUECK, K. 1972. As Florestas da América do Sul. São Paulo, Editorial Polígono. p.466. Tradução por H. Reichardt.

Instituto Estadual de Floresta do Rio de Janeiro - IEF. 2008. Programa RPPNRJ. RPPN Campo Escoteiro Geraldo Hugo Nunes, Rio de Janeiro.

International Union for Conservation of Nature and Natural Resources IUCN. 2008a. Table 5: Threatened species in each country (totals by taxonomic group). http://www.iucnredlist.org/amphibians (último acesso em: 02/04/2009).

International Union for Conservation of Nature and Natural Resources IUCN. 2008b. Table 3a. Status category summary by major taxonomic group (animals). http://www.iucnredlist.org/amphibians. (último acesso em: 30/03/2009).

International Union for Conservation of Nature and Natural Resources - IUCN. 2009. GAA Global Amphibian Assessment. http://www.iucnredlist.org/ amphibians. (último acesso em 19/12/2009).

LEWINSOHN, T.M. \& PRADO, P.I. 2005. Biodiversidade brasileira: síntese do estado atual do conhecimento. Campinas, Unicamp.
MORELLATO, L.P. \& HADDAD, C.F.B. 2000. Introduction: the Brazilian Atlantic Forest. Biotropica 32(4b):786-792.

PIMENTA, B.V.S., CRUZ, C.A.G., \& DIXO, M. 2002. Geographic distribution: Chiasmocleis carvalhoi. Herpet. Rev. 33:219

RANTA, P., BLOM, T., NIEMELÄ, J., JOENSUU, E., \& SIITONEN, M. 1998. The fragmented Atlantic rain forest of Brazil: size, shape and distribution of forest fragments. Biodiv. Conserv. 7:385-403.

RIBEIRO, M.C., METZGER, J.P., MARTENSEN, A.C., PONZONI, F.J., \& HIROTA, M.M. 2009. The Brazilian Atlantic Forest: How much is left, and how is the remaining forest distributed? Implications for conservation. Biol. Conserv. 142:1141-1153.

RODRIGUES, M.T. 2005. Conservação dos répteis brasileiros: os desafios para um país megadiverso. Megadiversidade 1(1):87-94.

SALLES, R.O.L., WEBER, L.N. \& SIVA-SOARES, T. 2009. Amphibia, Parque Natural Municipal da Taquara, municipality of Duque de Caxias, state of Rio de Janeiro, Southeastern Brasil. Check List: J. Spec. Lists Distrib. 5(4):840-854.

Sociedade Brasileira de Herpetologia - SBH. 2010. Brazilian amphibians. http://www.sbherpetologia.org.br. (último acesso em: 20/05/2010).

SILVA-SOARES, T., COSTA, P.N. \& FERREIRA, R.B. 2009. Geographic distribution: Chiasmocleis carvalhoi. Herpe. Rev. 40(1):107.

SILVANO, D.L. \& SEGALLA, M.V. 2005. Conservação de anfíbios no Brasil. Megadiversidade. 1(1):80-86.

STUART, S.N., HOFFMANN, M., CHANSON, J.S., COX, N.A., BERRIDGE, R.J., RAMANI P. \& YOUNG., B.E. 2008. Threatened amphibians of the world. Lynix Edicions, Barcelona.

União dos Escoteiros do Brasil. Campo Escola - História do Campo Escola. Rio de Janeiro. http://www.uebrj.org.br/ceghnHist.php. (último acesso em: 30/03/2009).

VINCIPROVA, G. \& GARCIA, P.C.A. 2002. Anfíbios. In Lista das espécies da fauna ameaçadas de extinção no Rio Grande do Sul. Decreto No 41.672 , de 10 de junho de 2002. (A.A.B. MARQUES, C.S. FONTANA, E. VÉLEZ, G.A. BENCKE, M. SCHNEIDER \& R.E. REIS). FZB/ MCTPUCRS/PANGEA, Porto Alegre, p.40. (Publicações avulsas FZB, 11). 


\section{Apêndice 1}

Brachycephalidae: Brachycephalus didactylus (ZUFRJ 2311, 2312, 2326); Bufonidae: Dendrophryniscus brevipollicatus (ZUFRJ 2285, 2332, 2431), Rhinella hoogmoedi (ZUFRJ 2286, 2386, 3402), Rhinella ornata (ZUFRJ 10815, 3278); Craugastoridae: Haddadus binotatus (ZUFRJ10808, 10809, 10515); Cycloramphidae: Thoropa miliaris (ZUFRJ 10819, 10820, 10821), Zachaenus parvulus (ZUFRJ 2334, 3397); Hemiphractidae: Flectonotus goeldii (ZUFRJ 3285); Hylidae: Aparasphenodon brunoi (ZUFRJ 10509, 2207, 2208, 3087), Dendropsophus anceps (MNRJ 56158), Dendropsophus berthalutzae (ZUFRJ 5266, 5267, 5268, 5269), Dendropsophus bipunctatus (ZUFRJ 104420, 10443, 2271, 7478), Dendropsophus elegans (ZUFRJ 10816, 10817, 10750, 10519), Dendropsophus giesleri (ZUFRJ 2245, 2246, 2715, 3082), Dendropsophus meridianus (ZUFRJ 10511, 10512, 2736, 7477), Dendropsophus minutus (ZUFRJ 2222, 10752, 11080, 11081), Dendropsophus pseudomeridianus (ZUFRJ 6757, 6759, 7128, 11063), Dendropsophus seniculus (ZUFRJ 10507, 10508, 3088, 3136), Hypsiboas albomarginatus (ZUFRJ 10441, 2220), Hypsiboas faber (ZUFRJ 10759, 10760, 10533, 11082), Hypsiboas semilineatus (ZUFRJ 10824, 10825, 10757, 10528), Phyllomedusa rohdei (ZUFRJ 10519, 7526), Scinax alter (ZUFRJ 2725, 10834, 10798), Scinax argyreornatus (ZUFRJ 2232, 6982, 10755, 10829), Scinax cuspidatus (ZUFRJ 7124, 7364, 7488), Scinax cf. eurydice (ZUFRJ 3282, 3283), Scinax humilis (ZUFRJ 2242, 10513, 10754, 10830), Scinax similis (ZUFRJ 10417, 10758), Sphaenorhynchus planicola (ZUFRJ 7673, 10522, 10800, 10801), Trachycephalus mesophaeus (ZUFRJ 3132, 10813); Leiuperidae Physalaemus signifer (ZUFRJ 2306, 10748, 10818); Leptodactylidae: Leptodactylus fuscus (ZUFRJ 2092, 10527, 10814), Leptodactylus marmoratus (ZUFRJ 10535), Leptodactylus latrans (ZUFRJ 10526, 10745, 10746), Leptodactylus spixi (ZUFRJ 3089, 10516, 10531, 10532); Microhylidae: Myersiella microps (ZUFRJ 2349, 2425, 2882), Chiasmocleis carvalhoi (ZUFRJ 2294, 2295, 2296, 2297); Euparkerella cochranae (ZUFRJ 2589, 3400, 10804, 10805). 